\title{
AFS dynamic evolution during the emergence of an active region
}

\author{
D. Spadaro ${ }^{1}$, S. Billotta ${ }^{1}$, L. Contarino ${ }^{1}$, P. Romano ${ }^{2}$, and F. Zuccarello ${ }^{2}$ \\ ${ }^{1}$ INAF - Osservatorio Astrofisico di Catania, Via S. Sofia 78, 95125 Catania, Italy \\ e-mail: dspadaro@ct.astro.it \\ 2 Dipartimento di Fisica e Astronomia - Sezione Astrofisica, Università di Catania, Via S. Sofia 78, 95125 Catania, Italy
}

Received 1 April 2004 / Accepted 25 May 2004

\begin{abstract}
Using data acquired during an observational campaign carried out at the THEMIS telescope in IPM mode, coordinated with other ground- and space-based instruments (IOACT, TRACE, EIT/SOHO, MDI/SOHO), we have analyzed the first evolutionary phases of a recurrent active region (NOAA 10050), in order to study the morphology and dynamics of its magnetic structures during their emergence and early development. The main result obtained from this analysis concerns the dynamic evolution of the arch filament system (AFS) crossing the polarity inversion line: the line of sight velocities determined from Doppler measurements confirm that the loops forming the AFS show an upward motion at their tops and a downward motion at their extremities, but also indicate that the upward motion decreases while the active region develops. Moreover, it has been found that, within the limits of the temporal cadence and spatial resolution of the instruments used, the first evidence of the active region formation is initially observed in the transition region and lower corona, and later on (i.e. after about $6 \mathrm{~h}$ ) in the inner layers (chromosphere and photosphere). Another interesting result concerns the analysis of the magnetograms, indicating that the initial increase in the magnetic flux seems to be synchronous with the appearance od the active region appearance in the transition region and lower corona, and that the rate of increase of the magnetic flux during the formation of the active region is not constant, but is steeper at the beginning (i.e. during the first $150 \mathrm{~h}$ ) than in the following period. All these results may indicate the presence of some mechanism that decelerates the magnetic flux emergence as more and more flux tubes rise towards higher atmospheric layers. Finally, we would like to stress the observed asymmetries between the preceding and the following sides of NOAA 10050: the p-side is more extented than the f-side, the p-side moves forward from the initial outbreak position much faster than the f-side recedes; the AFS f-side exhibits higher downflows than the p-side.
\end{abstract}

Key words. Sun: activity - sunspots - Sun: magnetic fields - Sun: filaments

\section{Introduction}

The birth and evolution of new active regions on the solar surface is a complex event caused by the emergence of a bundle of magnetic flux tubes which form at the bottom of, or just below, the convection zone where the dynamo operates (Parker 1993), rise due to their buoyancy and eventually break through the photosphere, expanding outwards. This highly organized physical process starting in the subphotospheric layers involves practically all the upper solar atmosphere, from the photosphere to the corona, where active regions appear, evolve and decay on time scales ranging from about a day to several months. The emergence of active regions brings a huge amount of magnetic flux to the photosphere, so as to dominate the large-scale structure of the magnetic fields on the Sun (for a recent review on this argument, see, e.g., van Driel-Gesztelyi 2002). The detailed study of the morphology, configuration and distribution of the emerging magnetic flux during the formation of solar active regions is important for gaining information on the origin and subsequent emersion of magnetic flux tubes, with significant implications for the understanding of the operation of the solar dynamo (van Driel-Gesztelyi 2002). It is also important to set up a criterion to establish, from the characteristic properties of its first phases, whether an active region will have a complete evolution (with a typical lifetime of $\sim 1-2$ months), or will diffuse in a short time (1-4 days). Note that this point is still open.

The first phases of young active regions are characterized, in particular, by a series of plasma flows and motions of the areas with stronger magnetic field, related to the dynamics of the emerging magnetic flux tubes (e.g., Zwaan 1985; Priest 1987). Clearly, many rearrangements of the magnetic field topology occur during this dynamic period. Strous et al. (1996), for instance, studied the horizontal dynamics of granules, pores, sunspots and facular elements in an active region (NOAA 5617) by tracking individual structures through the field of view, finding velocities of order $1 \mathrm{~km} \mathrm{~s}^{-1}$. Strous \& Zwaan (1999) also found that the magnetic flux emergence in the growing active region shows an intricate fine structure, characterized by upflows and downflows of $10-30 \mathrm{~km} \mathrm{~s}^{-1}$ coincident with small-scale emergence events such as rising loops in the chromosphere, while the photospheric footpoints of the magnetic flux tubes move apart with velocities of $\sim 1 \mathrm{~km} \mathrm{~s}^{-1}$. Such events, which imply the intrusion of low atmosphere plasma into the corona, are globally defined as Arch Filament System (AFS). These systems can be observed over several 
days, while the individual arches last only a few tens of minutes. As the flux emerges, new arches are formed while the older ones expand in the atmosphere (Alissandrakis et al. 1990). On the other hand, hot loops are observed overlaying the AFS at coronal heights (Malherbe et al. 1998, and references therein).

Cauzzi et al. (1996), moreover, found asymmetries in the vertical plasma flows between the leading and following legs of the magnetic flux tubes, which could be due to plasma flows from one leg of a magnetic loop to the other; the observed asymmetries, obtained by averaging over the totality of the magnetic structures, range from 60 to $150 \mathrm{~m} \mathrm{~s}^{-1}$, showing a predominance of downflow in the preceding part of three young regions with respect to the following part.

In this context, we recently observed the initial evolutionary phases of some active regions with the aim of studying the morphology and dynamics of magnetic elements (pores, sunspots, AFS) during their emergence and development, trying to determine their characteristic lifetime, topology and velocity field. More specifically, we carried out high spatial resolution observations of young active regions with the THEMIS telescope (Arnaud et al. 1998) equipped with the IPM instrument (Cavallini 1998). Full-disc images taken at the INAF Catania Astrophysical Observatory in the centre of the $\mathrm{H} \alpha$ line and in its blue wing supported these observations, guiding in the selection of the targets to watch through the restricted field of view of THEMIS (Zuccarello et al. 2002). We also requested MDI/SOHO (Scherrer et al. 1995) support to get the relevant magnetograms. EIT/SOHO (Delaboudinière et al. 1995) synoptic images in the four wavelength bands $(171,195,284$ and $304 \AA$ ), as well as TRACE (Handy et al. 1999) images acquired at $171 \AA$, were obtained to study the morphology and evolution of the transition region and corona above the selected active regions.

Here we report on the analysis of the observations concerning the active region NOAA 10050, which we selected from our dataset. This region was observed to appear in the upper solar atmosphere on July 26, 2002, at 01:00 UT; the first record of the relevant sunspot group is instead at 14:15 UT, with heliographic coordinates S07, E41 (Solar Geophyisical Data, July 2002). This region developed considerably and was present on the solar surface for more than three rotations of the Sun, before completely decaying. Hence it is an interesting object for the purpose of our investigation.

\section{Observations and description of NOAA 10050 global morphology}

We observed the emerging Active Region NOAA 10050 from July 26 to August 2, 2002, using data acquired by several instruments (THEMIS/IPM, INAF-OACT, SOHO/EIT, SOHO/MDI, TRACE). Figure 1 shows the data coverage for each instrument used.

The images obtained by the Telescope Heliographique pour l'Étude du Magnetisme et des Instabilites Solaires (THEMIS) operating in IPM mode were acquired in 18 spectral points,

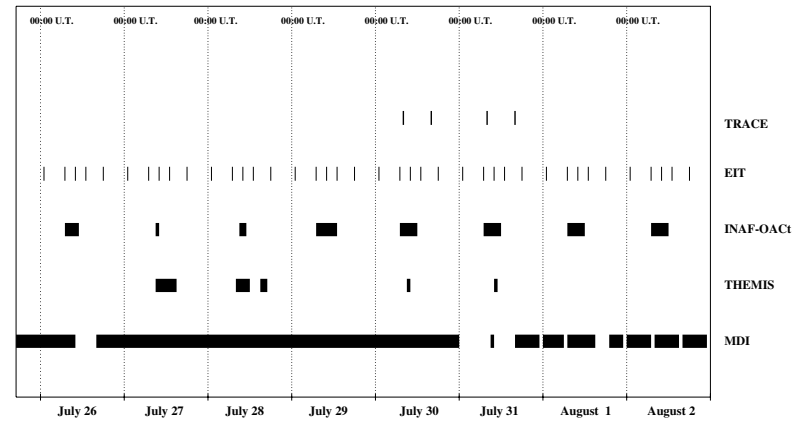

Fig. 1. Data coverage for the different instruments from July 26 to August 2, 2002.

11 along the profile of the $\mathrm{H} \alpha$ line $(\lambda=6562.808 \AA$, $+/-0.25 \AA$, $+/-0.50 \AA,+/-0.75 \AA,+/-1.00 \AA,+/-1.50 \AA$, with an exposure time of $300 \mathrm{~ms})$ and 7 along the Fe I line $(\lambda=5575.960 \AA$, $+/-0.040 \AA,+/-0.060 \AA,+/-0.090 \AA$, with an exposure time of $500 \mathrm{~ms}$ ). At the same time broad band images centered at $5380.960 \AA$ were acquired. The period employed to acquire the images in the 18 spectral points is $\sim 1 \mathrm{~min}$. The temporal resolution between two images is $\sim 3 \mathrm{~s}$ and the spatial resolution ranges from $\sim 0.2 \operatorname{arcsec}$ (best case) to $\sim 0.4 \operatorname{arcsec}$ (worst case).

During the whole observing campaign full disc images were acquired at INAF-Catania Astrophysical Observatory with a cadence of 30 minutes in the centre of the $\mathrm{H} \alpha$ line $(6563 \AA)$ and in its blue wing $(6568 \AA)$ with a pass band of $0.25 \AA$.

To study the magnetic field configuration and evolution of NOAA 10050 we used full disc magnetograms acquired by MDI/SOHO from 15:59 UT on July 25 to 23:59 UT on August 2 with an average time cadence of $1 \mathrm{~min}$ and a spatial resolution of 4 arcsec. Synoptic full disc images at $171 \AA$, $195 \AA, 284 \AA$ and $304 \AA$ were acquired by EIT/SOHO each day at 01:00, 07:00, 10:00, 13:00 and 18:00 UT, while TRACE observed the active region NOAA 10050 at $171 \AA$, on July 30 and 31 , during two short time intervals (08:30-08:45 and 15:50-16:00 UT).

No sign of NOAA 10050 was yet visible in the photospheric images acquired at the INAF-Catania Astrophysical Observatory on July 26, at 6:52 UT (see Fig. 2a). For completeness, we stress that the active region was not visible even in the WL image acquired by MDI on July 26, at 9:36 UT. The appearance of the region was recorded on July 27, 2002 (Fig. 2b). The sequence in Fig. 2 shows that the morphology of the region significantly changed from July 27 to August 2. Initially, it appears very compact, with an extented preceding spot, two small following spots and some pores. On the next day, July 28 (Fig. 2c), the morphology becomes more complex, the longitudinal extension increases and new spots and pores appear between the preceding spot and the following ones. The increasing complexity of the structure and its longitudinal extension seem to reach a maximum on July 31. On August 1 and 2, the active region is near the limb and its morphology does not exhibit significant variations.

The appearance of NOAA 10050 in the $\mathrm{H} \alpha$ sequence (Fig. 3) acquired at the INAF-Catania Astrophysical Observatory is first recorded on July 26 at 7:13 UT as a weak 


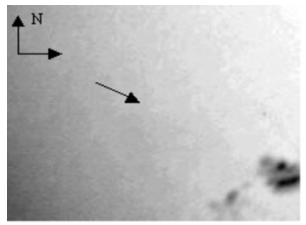

(a) 26/7

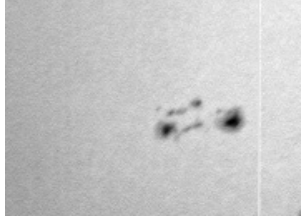

(c) $28 / 7$

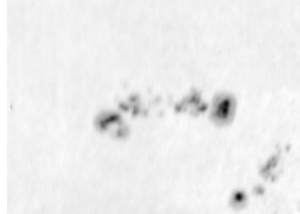

(e) $31 / 7$

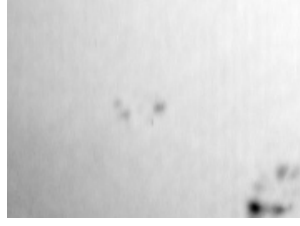

(b) $27 / 7$

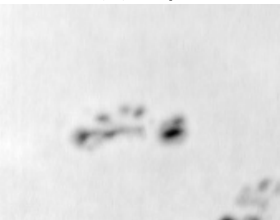

(d) $29 / 7$

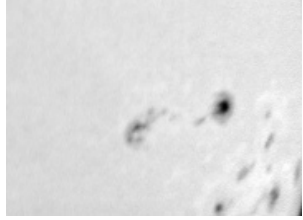

(f) $2 / 8$
Fig. 2. Photospheric daily images acquired at the INAF-Catania Astrophysical Observatory from July 26 a) to August 2 f). The field of view is $\sim 310000 \times 262000 \mathrm{~km}^{2}$. The arrow in image a) indicates the site where the sunspot group appears, as deduced from the magnetogram shown in Fig. 4a. In this figure and in Figs. 3 and 4 we omit the images of $30 / 7$ and $1 / 8$ because there are no significant changes in the active region configuration.

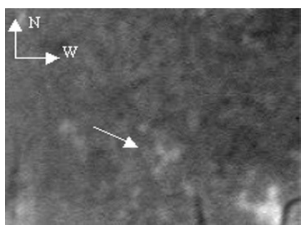

(a) $26 / 7$

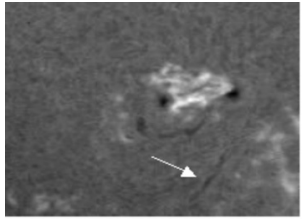

(c) $28 / 7$

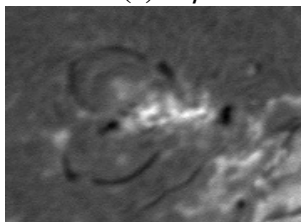

(e) $31 / 7$

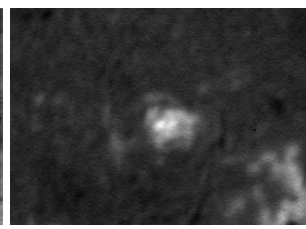

(b) $27 / 7$

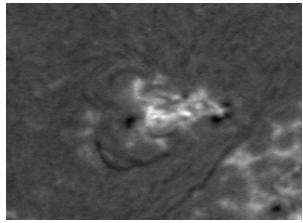

(d) 29/7

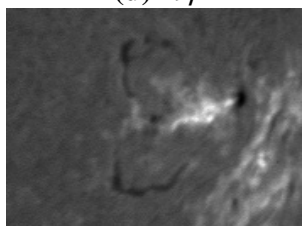

(f) $2 / 8$
Fig. 3. Chromospheric daily images acquired at INAF-Catania Astrophysical Observatory from July 26 a) to August 2 f). The field of view is $\sim 310000 \times 262000 \mathrm{~km}^{2}$. The arrows indicate the location where NOAA 10050 appears a) and the filament described in the relevant text c).

brightness increase. Note that earlier $\mathrm{H} \alpha$ images provided by the Global High Resolution $\mathrm{H} \alpha$ Network (see Zuccarello et al. 2002) do not show evidence of NOAA 10050. On July 27 it is already a compact facular structure, and the following day there is a rapid change in its morphology, with the appearence

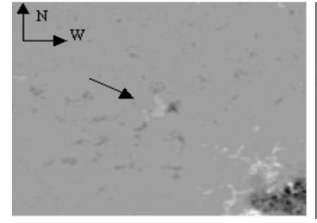

(a) $26 / 7$

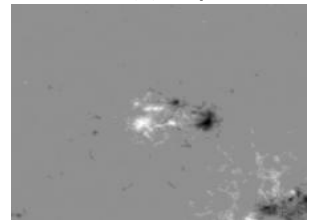

(c) $28 / 7$

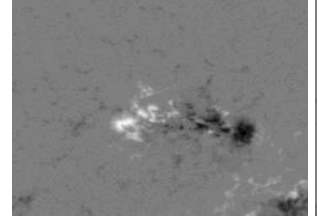

(e) $31 / 7$

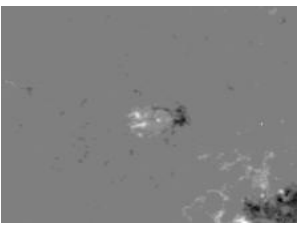

(b) $27 / 7$

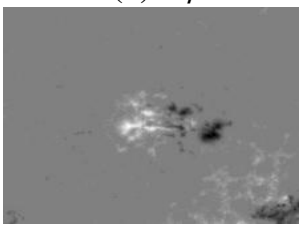

(d) $29 / 7$

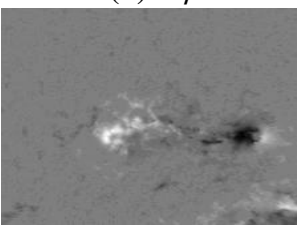

(f) $2 / 8$
Fig. 4. Daily magnetograms acquired by MDI at 8:00 UT from July 26 a) to August $2 \mathbf{f}$ ). The field of view is $\sim 310000 \times 262000 \mathrm{~km}^{2}$.

of an AFS between the two major spots and a large filament in the South part of the active region. The formation of a filament in the region between AR 10050 and AR 10044 is also worth noting (see the arrow in Fig. 3c), which disappears on August 2. On July 29, a new large external filament in the Northern part of the active region is observed. This filament is more evident on July 31 and remains until August 2.

Figure 4 shows a sequence of magnetograms acquired by MDI from July 26 to August 2, 2002. The emergence of new magnetic flux takes place on July 26 (S07, E41, see the arrow in Fig. 4a) and increases considerably during the transit by NOAA 10050 of the solar disc. We can see that the magnetic polarities are compact (Fig. 4b), then they increase initially in scale and intensity, significantly departing from each other.

It is worth noting that the active region already appears in the images acquired by EIT/SOHO in the He II $304 \AA$ band on July 26 at 1:00 UT as a small bright structure (see the arrow in Fig. 5). It is also evident in the images nearly simultaneously acquired in the other three EIT bands, even if these images have a lower contrast. Figure 6 shows two images acquired at $195 \AA$ by EIT/SOHO on July 26 and 31 . The increase of coronal emission is clearly visible on July 26 (see the arrow in Fig. 6a). In the subsequent days, the active region evolves considerably and several loop systems appear.

Therefore, it is evident that the evolution of the active region NOAA 10050 was very complex and rapid during its first transit of the solar disc (Figs. 2, 3, 6). Moreover it showed an intense flare activity (GOES-8 recorded $16 \mathrm{C}$-class flare events during this time interval). 


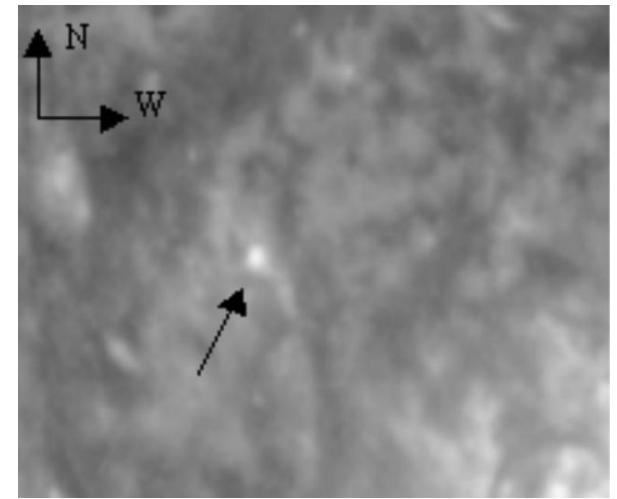

Fig. 5. Image acquired by EIT/SOHO in the HeII $304 \AA$ line at 1:00 UT on July 26 . The field of view is $\sim 310000 \times 262000 \mathrm{~km}^{2}$.

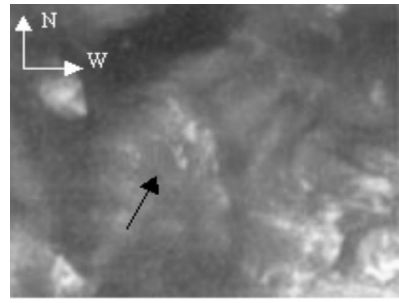

(a) $26 / 7$

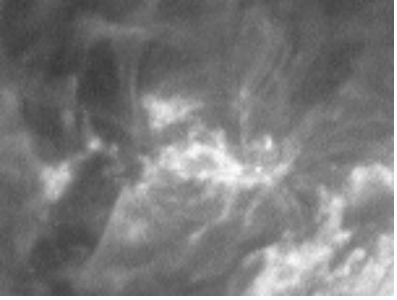

(b) $31 / 7$

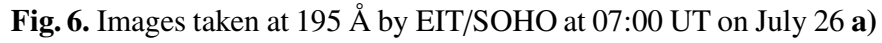
and July 31 b). The field of view is the same as in Fig. $5(\sim 310000 \times$ $\left.262000 \mathrm{~km}^{2}\right)$.

\section{Data analysis}

\section{1. $M D I$}

To study the rate of emergence of magnetic flux in NOAA 10050, we selected one MDI full disc magnetogram every hour, from 15:59 UT on July 25 to 23:59 UT on August 2 , 2002. For each magnetogram we limited our analysis to a subfield of $130 \times 65$ pixels $\left(182000 \times 91000 \mathrm{~km}^{2}\right)$ centered on the active region, and we determined both the positive and negative emerging magnetic flux, taking into account the corrections required by the projection effects. We also subtracted the background magnetic flux by measuring the average magnetic flux in a small area of $30 \times 10$ pixels $\left(42000 \times 14000 \mathrm{~km}^{2}\right)$, close to the active region (Chae 2001).

\subsection{THEMIS/IPM}

The high spatial resolution images acquired by the THEMIS telescope allowed us to study the first phase of evolution of the active region in more detail. During each observing run we sequentially took images along the $\mathrm{H} \alpha$ and the FeI lines in different fields of view, in order to scan the whole area where the active region appeared several times. The analyzed THEMIS dataset is reported in Table 1.

We corrected the THEMIS/IPM data applying the standard dark current and flat field corrections (e.g., Contarino et al. 2003). The flat field images were acquired while the telescope was changing its field of view so as to describe an ellipse on the Sun. We averaged all the images relevant to each spectral point, in order to take into account the detector sensitivity pattern and the optical path effects for different wavelengths. Then we divided each raw monochromatic image by the corresponding flat field image. An analogous procedure was applied to the broad band images. We discarded the unused areas of the detector by restricting our analysis to a sub-field of $34 \times 34 \mathrm{arcsec}^{2}$.

We reconstructed the profiles of the FeI and $\mathrm{H} \alpha$ lines in each spatial pixel by fitting the corresponding signals obtained in the monochromatic images with a linear background and a Gaussian shaped line:

$I_{j}=A_{0} \exp \left(-\left(\frac{\lambda_{k}-A_{1}}{A_{2}}\right)^{2}\right)+A_{3}+A_{4} \lambda_{k}$

where $I_{j}$ is the observed count rate in pixel $j$ at wavelength $\lambda_{k}$, $A_{0}$ the height of the Gaussian, $A_{1}$ the centroid wavelength of the Gaussian, $A_{2}$ the $1 / \mathrm{e}$ half width of the Gaussian, $A_{3}$ the constant term and $A_{4}$ the linear term. Values of the parameters $A_{0}$, $A_{1}, A_{2}, A_{3}$ and $A_{4}$ were obtained by the maximum likelihood method.

The values of velocity along the line of sight in photosphere and chromosphere have been deduced from the Doppler shift of the centroid of the line profiles in each spatial point with respect to the median of the centroid in the whole field of view. The median is used as wavelength reference, since there is no absolute wavelength reference in IPM. We estimated the uncertainty affecting the velocity measurements considering the standard deviation of the centroids of the line profiles estimated in all points of the whole field of view. The thus estimated errors in the velocity are $\pm 0.2 \mathrm{~km} \mathrm{~s}^{-1}$ and $\pm 1 \mathrm{~km} \mathrm{~s}^{-1}$ for the Fe I and $\mathrm{H} \alpha$ line, respectively.

To determine the horizontal photospheric velocity field we applied the cross correlation procedure described by Kitay et al. (1997) to broad band images. The field of view of our images was $340 \times 340$ pixels. We divided the images into subfields of $15 \times 15$ pixels (we did not take into account both the first 15 and the last 20 rows and columns in the field of view in order to exclude the errors introduced by the alignment). Each subfield is represented by a pair of integers $\left(x_{0}, y_{0}\right)$, where $x_{0}$ is the left column number and $y_{0}$ is the bottom row number.

The cross-correlation between two images observed at different times ( $t$ and $t+\delta t$ ), calculated for each subfield, is given by:

$C C\left(\delta x, \delta y, x_{0}, y_{0}\right)=$

$\frac{\iint_{S} I_{t}(x, y) I_{t+\delta t}(x+\delta x, y+\delta y) \mathrm{d} x \mathrm{~d} y}{\sqrt{\iint_{S} I_{t}^{2}(x, y) \mathrm{d} x \mathrm{~d} y} \sqrt{\iint_{S} I_{t+\delta t^{2}}(x+\delta x, y+\delta y) \mathrm{d} x \mathrm{~d} y}}$

where $C C$ is the coefficient of cross-correlation, $I_{t}$ and $I_{t+\delta t}$ are the intensities at $t$ and $t+\delta t,(\delta x, \delta y)$ is the relative displacement of the image at $t+\delta t$ with respect to the image at $t$, and $S$ is the area of the considered subfield. The pair $(\delta x, \delta y)$ corresponding to the highest $C C$ gives the displacement vector $(\delta \boldsymbol{r})$ of the subfield during $\delta t$. The corresponding velocity is determined as $\boldsymbol{v}=\delta \boldsymbol{r} / \delta t$. We calculated the cross correlation between consecutive images for all the $n$ images acquired during the observing time $T$, obtaining $(n-1)$ displacements, $\delta \boldsymbol{r}_{i}$, for each subfield. 
Table 1. THEMIS data set.

\begin{tabular}{cccc}
\hline \hline Day & Observing time (UT) & Selected fields of view & Performed sequences \\
\hline July 27 & $08: 47-10: 19$ & 6 & 15 \\
July 28 & $07: 54-11: 53$ & 7 & 30 \\
July 30 & $09: 13-10: 31$ & 13 & 5 \\
July 31 & $10: 10-10: 50$ & 13 & 3 \\
\hline
\end{tabular}

Then, to increase the statistical accuracy of the derived velocities, we calculated the average of the displacements and the average velocity for each subfield during $T$ as:

$\langle\boldsymbol{v}\rangle_{n}=\frac{\sum_{i=1}^{n-1} \delta \boldsymbol{r}_{i}}{\sum_{i=1}^{n-1} \delta t_{i}}=\frac{\sum_{i=1}^{n-1} \delta \boldsymbol{r}_{i}}{T}$.

\section{Results}

\subsection{Magnetic flux}

Figure $7 \mathrm{a}$ reports both the positive (thin line) and negative (thick line) magnetic flux, in absolute value, as a function of time. The origin of the abscissa corresponds to 15:59 UT, on July 25. The interruptions in the magnetic flux profiles correspond to temporal gaps in the MDI observations. During the first $18 \mathrm{~h}$ we do not see any significant variation of the magnetic flux, which exhibits an average value of $2.5 \times 10^{22} \mathrm{Mx}$ in both polarities. $24 \mathrm{~h}$ after the beginning of the observing run we notice a steep increase in the magnetic flux. In $150 \mathrm{~h}$ we register an increase by $\sim 1.5 \times 10^{23} \mathrm{Mx}$ and $\sim 1.8 \times 10^{23} \mathrm{Mx}$ for the positive and negative magnetic flux, respectively. We also note an alternate prevalence of the two polarities. From July 31 to the end of the observations, we note a smoother increase of the global magnetic flux, whose values remain above $2 \times 10^{23} \mathrm{Mx}$, as well as an increase of the difference between the two magnetic polarities $\left(\sim 0.5 \times 10^{23} \mathrm{Mx}\right.$ at 23:59 UT, on August 2$)$.

To point out the first emergence of magnetic flux concentrations, we analyzed the magnetograms acquired from 15:59 UT, on July 25 , to 9:59 UT, on July 26 , in more detail. We computed the magnetic flux selecting only structures with a magnetic field larger than an absolute threshold value of $300 \mathrm{G}$, and report the results in Fig. $7 \mathrm{~b}$. We note a steep increase in the negative magnetic flux (thick line) around 1:00 UT on July 26, i.e. at the same time when we registered the first appearance of NOAA 10050 in the transition region and lower corona (see Sect. 2). A steep increase in the positive magnetic flux (thin line) also occurs about $6 \mathrm{~h}$ later.

This rapid and continuous emergence of magnetic flux from July 25 to August 2 is also accompanied by an asymmetric horizontal motion of both polarities. In fact, the displacements of the centre of symmetry of each polarity determined from MDI magnetograms (Fig. 8) show that the preceding (negative) polarity moves away from the inversion line more quickly than the following one. We determined an average velocity (in absolute value) of $\sim 0.15 \mathrm{~km} \mathrm{~s}^{-1}$ and of $\sim 0.08 \mathrm{~km} \mathrm{~s}^{-1}$ for the negative and positive polarities, respectively. This difference in the direction and values of velocities is in agreement with the hypothesis of the emergence of inclined flux tubes, as suggested

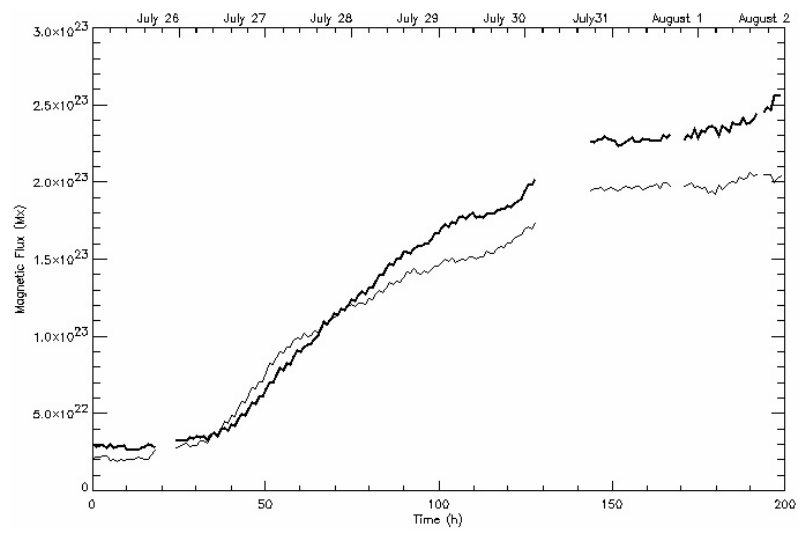

(a)

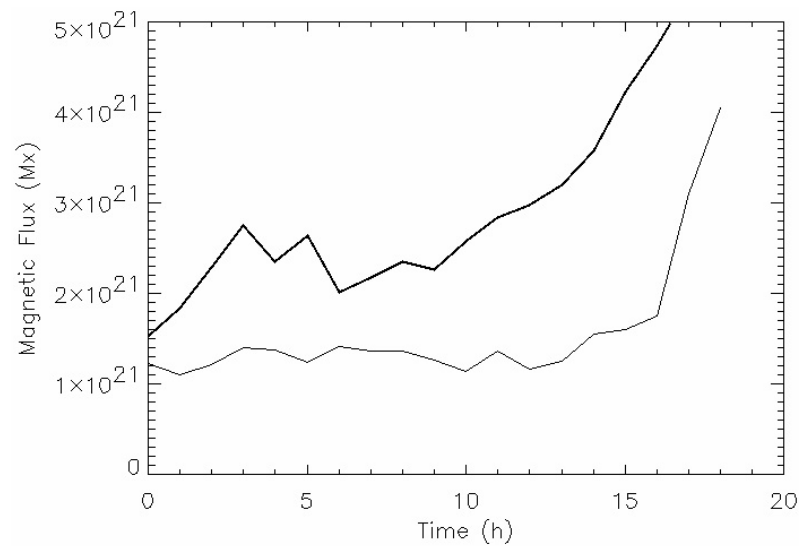

(b)

Fig. 7. a) Magnetic flux in NOAA 10050 from 15:59 UT on July 25 to 24:00 UT on August 2, 2002, as deduced from MDI full disk magnetograms. The thin and thick lines represent the positive and negative magnetic flux (in absolute value), respectively. The interruptions correspond to temporal gaps in the MDI observations. b) Magnetic flux variation in structures with a magnetic field larger than an absolute threshold value of $300 \mathrm{G}$ during the first $20 \mathrm{~h}$.

in previous studies (van Driel \& Petrovay 1990; Petrovay et al. 1990; Howard 1991; Caligari et al. 1995).

\subsection{Morphology and evolution}

We reconstructed images of the entire active region from each scan, both in broad band and in $\mathrm{H} \alpha$, adopting the crosscorrelation method between the overlapping areas of contiguous images at the same wavelength. Figure 9 reports some reconstructions of NOAA 10050 obtained from the observations carried out on July 27 and 28. The images in the centre of 


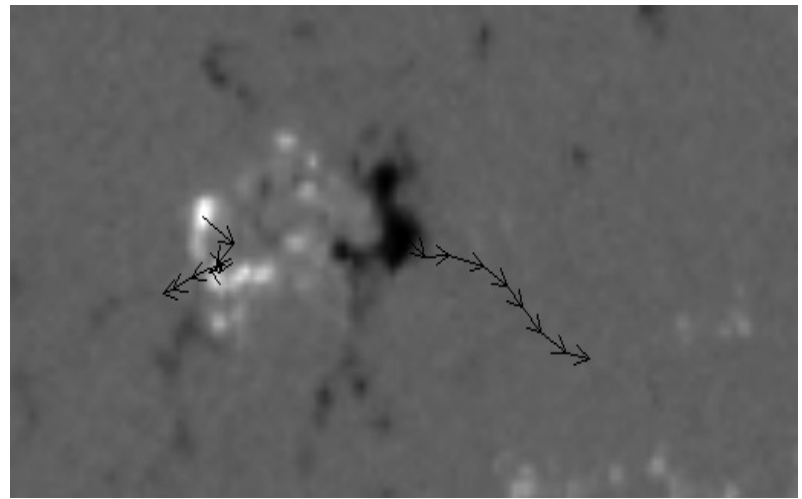

Fig. 8. MDI magnetogram taken at 23:58 UT on July 26. The arrows indicate the shift of the polarity centres of symmetry in the following days, till July 30 at 23:58 UT.

the $\mathrm{H} \alpha$ line show that the main characteristic of the early phases of formation of this active region is an arch filament system located between the two emerging magnetic polarities. This AFS is composed of tens of arch filaments (AF) grouped in bundles, approximately parallel and located across the polarity inversion line (PIL), with an average length of $\sim 20$ arcsec.

Figures 10 and 11 show images of a particular field of view, obtained at different times in the centre of the $\mathrm{H} \alpha$ line on July 27, when NOAA 10050 was growing. We can distinguish 7 AFs in Fig. 10b: 4 (named $\alpha, \beta, \gamma$ and $\delta$ ) seeming rather stable and 3 (named $\varepsilon, \phi$ and $\theta$ ) more dynamic. The last 3 AFs appear in the Eastern part of the active region, where the following positive magnetic component was emerging. In the photosphere, observed in the line of Fe I (5577.15 $\AA$ ) at 9:33 UT (see Fig. 10a) this area is characterized by some pores. Note, in particular, the formation of the $\mathrm{AF} \varepsilon$ in the time interval between 9:25 and 9:34 UT (Fig. 10b-1). This AF is not parallel to the others and seems to be rooted in a brightening region. At the same time, from 9:25 to 9:28 UT we also notice the formation of AFs $\phi$ and $\theta$ (Fig. 10b-e). Their absorption in the centre of the $\mathrm{H} \alpha$ line progressively spreads from East to West in the field of view, near the pre-existent $\mathrm{AF} \delta$.

Some AFs ( $\varepsilon$ and $\delta$ ) are not present in the $\mathrm{H} \alpha$ image taken at 9:54 UT (Fig. 11a). Therefore we deduce that they disappeared during the $20 \mathrm{~min}$ interval between the acquisition of the $\mathrm{H} \alpha$ image reported in Fig. 101 and of that in Fig. 11a. On the other hand, we note that $\mathrm{AF} \phi$ seems to re-form in the same place as before, during the interval 9:54-9:58 UT, while AF $\theta$ appears almost stable.

Moreover, there is a remarkable bundle of AFs in the bottom left of the field of view. It appears in all the $\mathrm{H} \alpha$ images reported in Figs. 10 and 11, without exhibiting a significant evolution during the time interval 9:25-9:58 UT.

On July 28 NOAA 10050 is already a complex and extended active region, and the magnetic flux is still emerging with a fast rate. We observe the AFS (Fig. 12b) in the area between the two major spots, where there are also some pores (Fig. 12a). The characteristics of this AFS are different with respect to the day before: its AFs are less numerous, with various dimensions, various orientations and a slower dynamic evolution. We are able to distinguish 4 AFs, which we
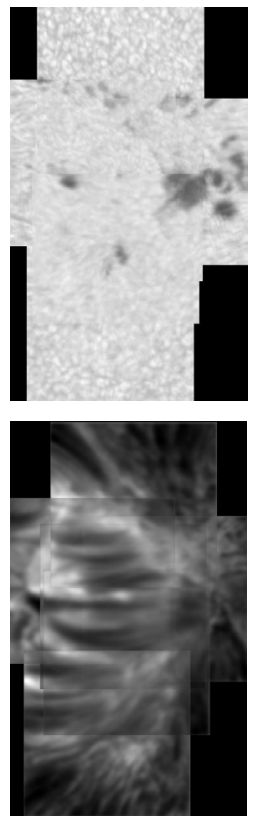

(a)
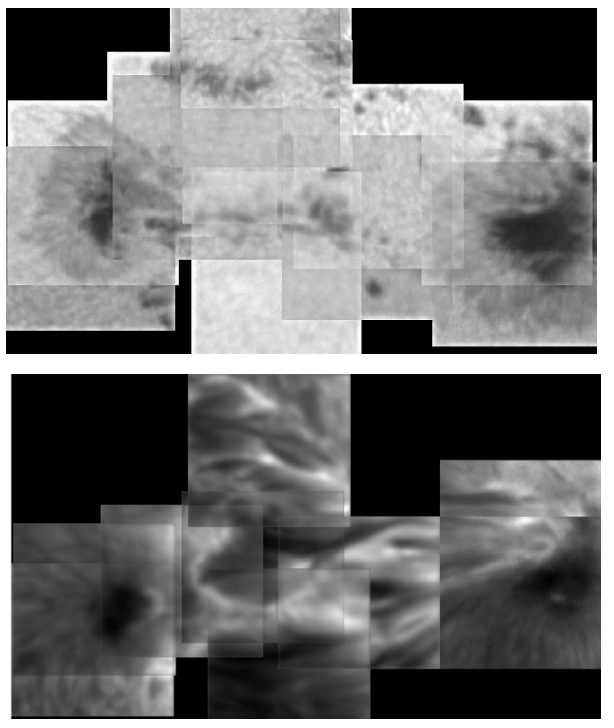

(b)

Fig. 9. Reconstructions of NOAA 10050 obtained from the images acquired by the THEMIS telescope in the continuum at $5380.96 \AA$ (top) and in the centre of the $\mathrm{H} \alpha$ line (bottom), on July 27 a) and 28 b), respectively. The field of view is $\sim 35000 \times 58000 \mathrm{~km}^{2}$ on July 27 and $\sim 83000 \times 45000 \mathrm{~km}^{2}$ on July 28 .

named $\kappa, \lambda, \mu$ and $v$, and some smaller ones indicated by the arrows in Fig. 12b. As we can see in Figs. 12b and 12c (taken with a time cadence of $3 \mathrm{~min}$ ), the smaller AFs show a faster morphological evolution than the bigger ones.

On July 30 all AFs still have various lengths and orientations, however they always appear in the area between the main sunspots, where new flux is emerging, as observed in MDI magnetograms.

The day after, in a small portion of the active region (Fig. 13a), the AFS seems to re-establish an homogeneous 


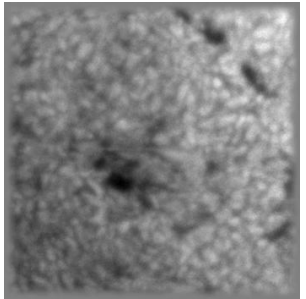

(a) 9:33 UT

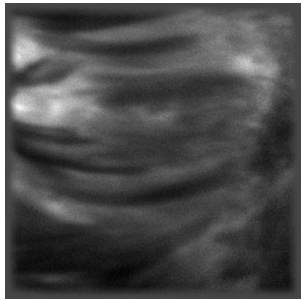

(c) 9:26 UT

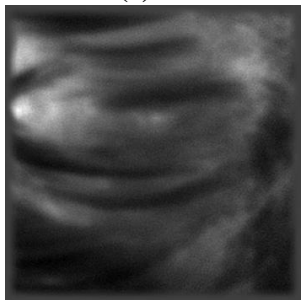

(e) 9:28 UT

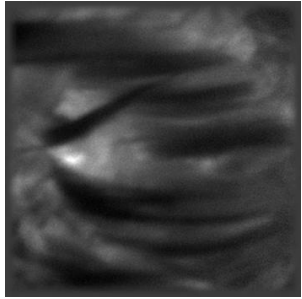

(g) 9:31 UT

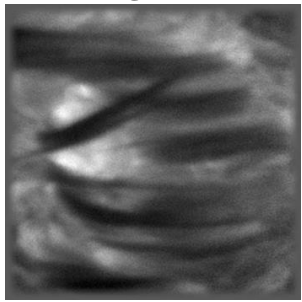

(i) 9:33 UT

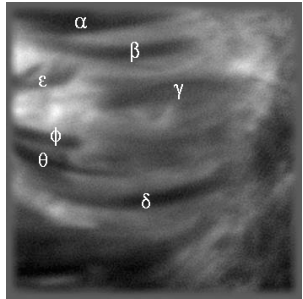

(b) 9:25 UT

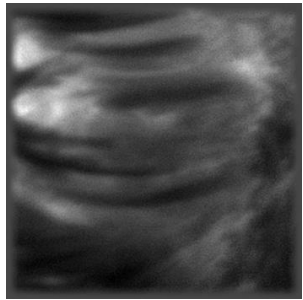

(d) 9:27 UT

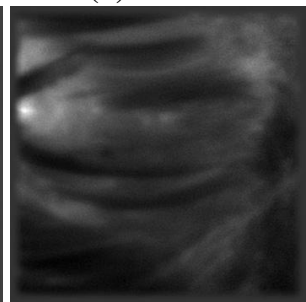

(f) 9:29 UT

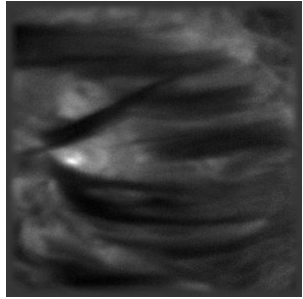

(h) 9:32 UT

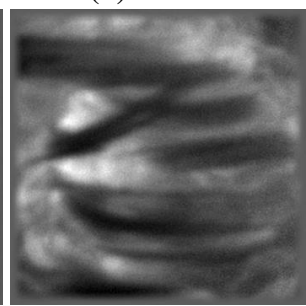

(l) 9:34 UT
Fig. 10. Image taken at $5577.15 \AA$ a) and a sequence of images of the same field of view taken at $6562.808 \AA$ b)-l) on July 27. In all images of this and the other figures. North is at the top, West on the right and the field of view is $\sim 24000 \times 24000 \mathrm{~km}^{2}$.

configuration and its AFs appear parallel, thin and close to each other (Fig. 13b).

We have also analyzed the images acquired at $171 \AA$ by TRACE on July 30 and 31. Figure 14 shows an image of NOAA 10050 acquired by TRACE at 8:33 UT on July 30. We note bright loop structures in the inner area of the active region, between the two major magnetic polarities (see enlarged view in the insert). These coronal structures seem to have the same morphology and orientation as the AFs observed in $\mathrm{H} \alpha$, with lengths ranging from 10 to 25 arcsec. During the short observing time (about $15 \mathrm{~min}$ ), they show no significant change.

\subsection{Velocity fields}

The study of the plasma velocity along the line of sight (los) deduced from the Doppler shift of the spectral lines allowed us to register some characteristics of the motions in the AFS, assuming that it is a collection of thin magnetic loops (e.g. Strous \& Zwaan 1999), with a very high opacity in the considered lines. It is worth noting that los velocities derived from line Doppler shifts are generally underestimated if the AFS is not optically thick (cf. Mein et al. 1996). Fig. 15 shows a los velocity map obtained from the analysis of the $\mathrm{H} \alpha$ profile in the area characterized by the AFS on July 27 at 9:32 UT. We plot the velocity contours every $2 \mathrm{~km} \mathrm{~s}^{-1}$ over the image taken in the centre of the $\mathrm{H} \alpha$ line. Thick and thin contours indicate positive (upward) and negative (downward) los plasma motion, respectively. We note that the top of each AF is characterized by a plasma positive velocity, which indicates upward motion of the entire AF structure, which rises in the chromosphere. At the same time, the ends of the AFs generally exhibit negative velocities, which can be interpreted as signatures of a downward plasma motion along the magnetic field lines at the footpoints of each AF.

Table 2 reports the maximum los upward velocity at the top of the AFs, as well as the maximum downward velocities at their ends, determined on July 27 and 28 . We note the highest value of $\sim 9 \mathrm{~km} \mathrm{~s}^{-1}$ at the top of AF $\alpha$, while the following end of $\mathrm{AF} \beta$ is characterized by the highest downward los velocity $\left(\sim-17 \mathrm{~km} \mathrm{~s}^{-1}\right)$. Moreover, all the AFs show an asymmetry in the los velocities at their ends: there is a stronger downflow in the following footpoints than in the preceding ones. In some cases the preceding end exhibits a slight upflow. This asymmetric behaviour is also evident on the other days of observation, even if the difference in los velocity between the preceding and the following ends decreases progressively.

The noticed asymmetry could be due in part to the inclination of the emerging AFs, so that the magnetic field lines in the following footpoints are closer to the vertical to the solar surface, enhancing the component of the velocity along the line of sight, while in the preceding ones they are more inclined with respect to the vertical, reducing the los velocities. This inclination of the AFs is similar to that deduced for the flux tubes rooted in the main magnetic polarities of the active region, according to the horizontal displacements of their footpoins (see Fig. 8). On the other hand, if we assume equal values of downflow velocities at the ends of the AFs, we can evaluate a lower limit for the angle between the preceding leg of each $\mathrm{AF}$ and the line of sight: $\alpha=\arccos \left(v_{\mathrm{f}} / v_{\mathrm{p}}\right)$, where $v_{\mathrm{f}}$ and $v_{\mathrm{p}}$ are the velocities of the following and preceding ends, respectively. For AFs $\alpha, \beta$ and $\gamma$ we find an inclination of $\sim 68^{\circ}, \sim 73^{\circ}$ and $\sim 67^{\circ}$, respectively. These angles of inclination are however rather large, so that the asymmetry between the following and preceding ends cannot be explained by geometric projection effects only, but concurrently by differences in the values of the flow velocity.

Another interesting point resulting from the maximum upward velocities measured at the top of the AFs for each observing day is the decrease whit time of the rising velocity of the AF structures, from $\sim 9 \mathrm{~km} \mathrm{~s}^{-1}$, on July 27 , to $\sim 2 \mathrm{~km} \mathrm{~s}^{-1}$, on July 31 (see Fig. 16). This systematic slowing down in the 


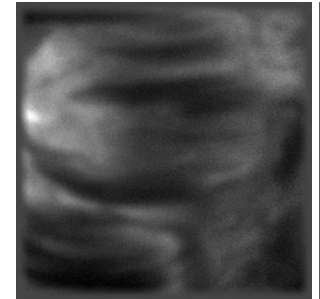

(a) 9:54 UT

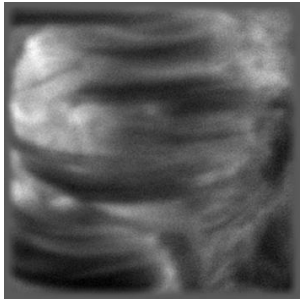

(b) 9:55 UT

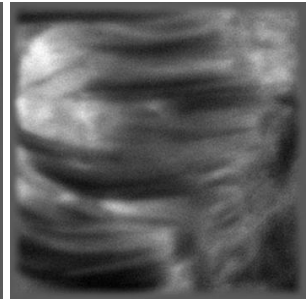

(c) 9:56 UT

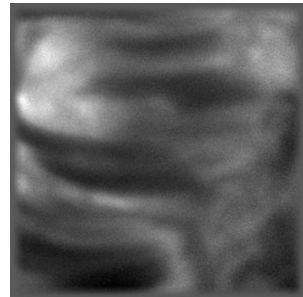

(d) 9:57 UT

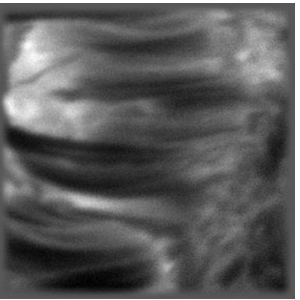

(e) 9:58 UT

Fig. 11. Sequence of images taken at $6562.808 \AA$ A on July 27 in a time interval following that of Fig. 10 .

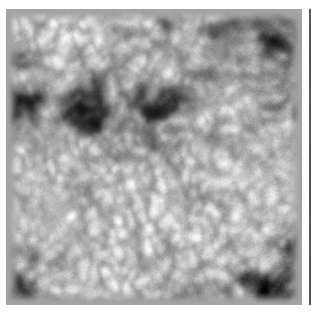

(a) 11:19 UT

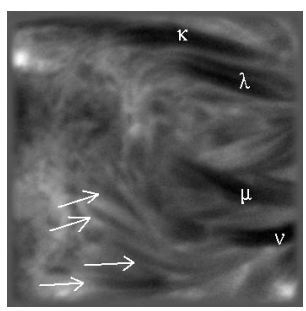

(b) 11:16 UT

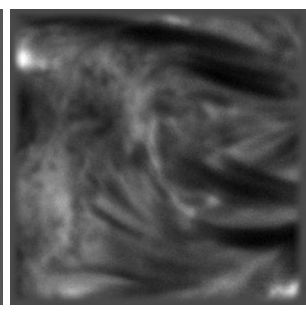

(c) 11:19 UT

Fig. 12. Image taken at $5577.15 \AA$ a) and two images of the same field of view taken at $6562.808 \AA$ b)-c) on July 28.

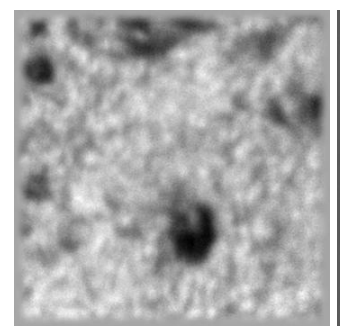

(a) 10:17 UT

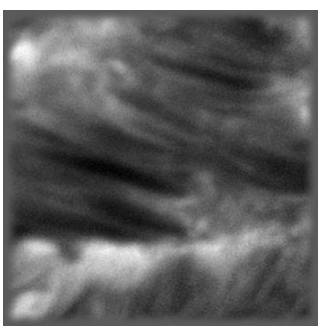

(b) 10:17 UT
Fig. 13. Images of the same field of view taken at $5577.15 \AA$ a) and at $6562.808 \AA$ A b) on July 31 .

upward motion of the new structures forming each day in the AFS might reflect some characteristics of the emerging magnetic flux in the active region. For istance, the overlaying older arches, not yet completely expanded in the higher atmosphere, could compress the newly forming ones while they rise in the chromosphere. This hypothesis is supported by the observation of bright coronal structures nearly coincident with the location and morphology of the examined AFS (see in Fig. 14 the image of NOAA 10050 acquired by TRACE). It is worth noting that even the los downward velocities at the footpoints of the AFs, as well as the differences in velocity between the preceding and following end, decrease progressively.

We studied in more detail the dynamic behaviour of some AFs, $\epsilon, \phi$ and $\theta$ (see Fig. 10), whose formation was discussed in Sect. 4.2 as occuring on July 27 in about 5 min. At the beginning, the upward los velocity measured at their top is $\sim 10 \mathrm{~km} \mathrm{~s}^{-1}$, gradually decreasing from the top to the ends. We also note a decrease in the top upward velocity to $\sim 4 \mathrm{~km} \mathrm{~s}^{-1}$ as the AFs form completely. This implies a significant slowing down in the rise already during the first phases of formation. Moreover, AF $\epsilon$ clearly appears to eject its plasma upward from the Eastern side, while a downward line of sight velocity of $\sim-20 \mathrm{~km} \mathrm{~s}^{-1}$ is measured in the bright (in $\mathrm{H} \alpha$ emission) region where this AF seems to be rooted.

To complete our analysis of the plasma velocities along the line of sight we used the Fe I line at $5575.96 \AA$, in order to evaluate their values at photospheric level. The results obtained, however, do not exhibit any peculiar pattern, because the differences in los velocity noticed among different areas or structures in the active region examined are within the estimated errors $\left(<0.2 \mathrm{~km} \mathrm{~s}^{-1}\right)$.

We also investigated the horizontal motions of the plasma near the photospheric footpoints of the considered arch structures, in order to have a complete picture of the dynamic situation characterizing the AFS. To this aim, we adopted the crosscorrelation procedure (see Sect. 3) in the analysis of the broad band images. We considered one data set for each day (July 27 and 28) relevant to the field of view where the AFS was present.

Figure 17a shows the map of the horizontal velocities (arrows) overplotted on the corresponding broad band image. The continuous lines in the image outline the edges of the overhanging AFs identified in the simultaneous $\mathrm{H} \alpha$ image (see Fig. 10b). 


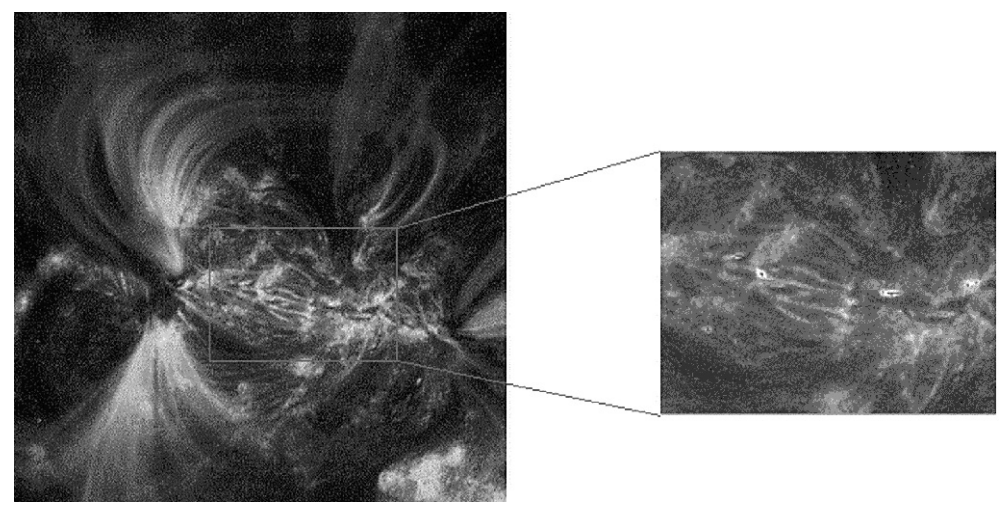

Fig. 14. Image of NOAA 10050 acquired by TRACE at $171 \AA$ on July 30,2002 . The field of view is $\sim 165000 \times 165000 \mathrm{~km}^{2}$. The insert shows an enlarged view of the inner region between the two major magnetic polarities (FOV $60000 \times 40000 \mathrm{~km}^{2}$ ).

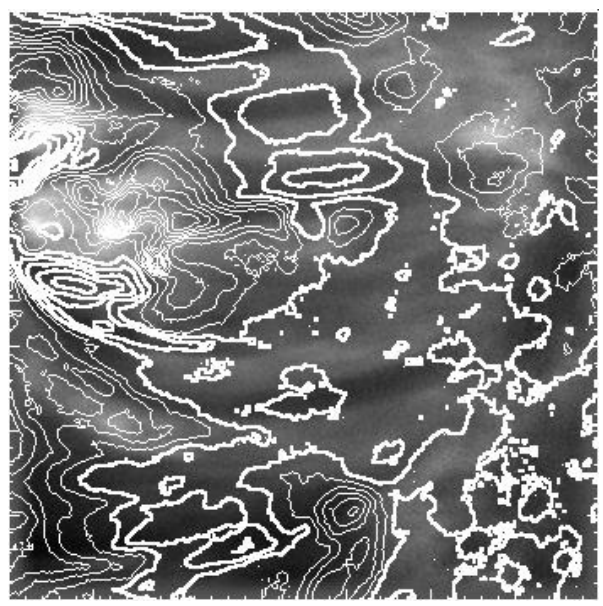

Fig. 15. Velocity map of the area characterized by the AFS and obtained using the $\mathrm{H} \alpha$ profile on July 27 at 9:32 UT. The map is plotted over an image acquired in the centre of the $\mathrm{H} \alpha$ line. North is at the top, West on the right. Thick contours indicate upward plasma motions, while thin contours indicate downward plasma motions. The velocity contours are drawn every $2 \mathrm{~km} \mathrm{~s}^{-1}$.

Figure 17b shows the analogous map obtained on July 28, with the AFs singled out in Fig. 12b. The average value calculated for the horizontal velocities is $\sim 3 \mathrm{~km} \mathrm{~s}^{-1}$. However, the maps do not indicate systematic motions in the region considered: the pattern appears random in general, suggesting a turbulent scenario for the rise of the magnetic flux tubes, at least at photospheric level. We only note a possible systematic motion at the footpoints of the AFs $\kappa$ and $\lambda$ (on July 28), which appear to be sheared counterclockwise with respect to the other end of the arch filaments, with a velocity $\sim 3.5 \mathrm{~km} \mathrm{~s}^{-1}$.

\section{Discussion and conclusions}

The aim of this paper is to give a contribution to the study of active region formation and evolution. Thus we have analyzed the first evolutionary phases of the recurrent active region NOAA 10050.

Even if the limits set by the time cadence and the spatial resolution of the instruments used must be taken into account, our analysis indicates that the first evidence of the emerging active region is observed in the EIT bands (forming in the transition region and lower corona); later on it appears in $\mathrm{H} \alpha$ (chromosphere) and white light (photosphere). These observations could imply that the manifestation of the emergence of an active region occurs first in the outer solar atmosphere and subsequently in the inner layers.

The magnetic flux shows a sudden, abrupt increase nearly simultaneous with the first manifestation of the active region in the transition region and lower corona. Subsequently the magnetic flux increase is slower.

The main characteristic of NOAA 10050 during the early phases of its development is an AFS located between the two emerging polarities. This structure represents the observational evidence of the hypothesis suggesting that $\Omega$-loops, when breaking through the photosphere, are broken into nearly parallel strands. The fragmentation takes place immediately below the photosphere and indicates the difficulty encountered by a flux tube to cross this layer (Van Driel-Gesztelyi 2002).

The arches of the AFS show an upward motion at their tops and generally downward motions at their extremities. The horizontal motions near the photospheric footpoints, conversely, do not show any systematic behaviour. Moreover, the values of both upflow and downflow velocities decrease during the evolution of the active region. While it is clear that upward motion on the top of the AFs is indicative of the buoyancy of the magnetic flux tubes and of their rise towards higher atmospheric levels, it is perhaps worthwhile to stress that the downward motion observed in the arch legs could be due to one (or a combination) of the following reasons: a) action of the Coriolis force on the rising flux tube; b) sliding motion associated with the Parker instability; c) remnant of a longitudinal flow in the original equilibrium (Moreno-Insertis 1997).

Taking into account these results, i.e., the decreasing upward motion of the AFS as the active region gets older, the simultaneous occurrence of the increase in the magnetic flux and the appearance of a bright structure in the transition region and lower corona, as well as the subsequent appearance of the active region features in the lower atmospheric layers, we can conclude that the thin flux tubes forming the $\Omega$-loop are initially characterized by a higher rising velocity, which eventually decreases. Therefore these observations may indicate the presence of some physical process which slows down 
Table 2. Maximum velocity at the top and at the ends of the AFs observed on July $27(\alpha, \beta, \gamma$ and $\delta)$ and July $28(\kappa, \lambda, \mu$ and $v)$.

\begin{tabular}{cccc}
\hline \hline AF & $\begin{array}{c}\text { Velocity of the } \\
\text { following end }\left(\mathrm{km} \mathrm{s}^{-1}\right)\end{array}$ & $\begin{array}{c}\text { Velocity of the } \\
\text { preceding end }\left(\mathrm{km} \mathrm{s}^{-1}\right)\end{array}$ & $\begin{array}{c}\text { Velocity } \\
\text { of the top }\left(\mathrm{km} \mathrm{s}^{-1}\right)\end{array}$ \\
\hline$\alpha$ & -16 & -6 & 9 \\
$\beta$ & -17 & -5 & 6 \\
$\gamma$ & -13 & -5 & 7 \\
$\delta$ & -9 & 1 & 6 \\
$\kappa$ & -3 & 1 & 3 \\
$\lambda$ & -5 & 0 & 3 \\
$\mu$ & -5 & -3 & 3 \\
$\nu$ & -6 & 1 & 3 \\
\hline
\end{tabular}

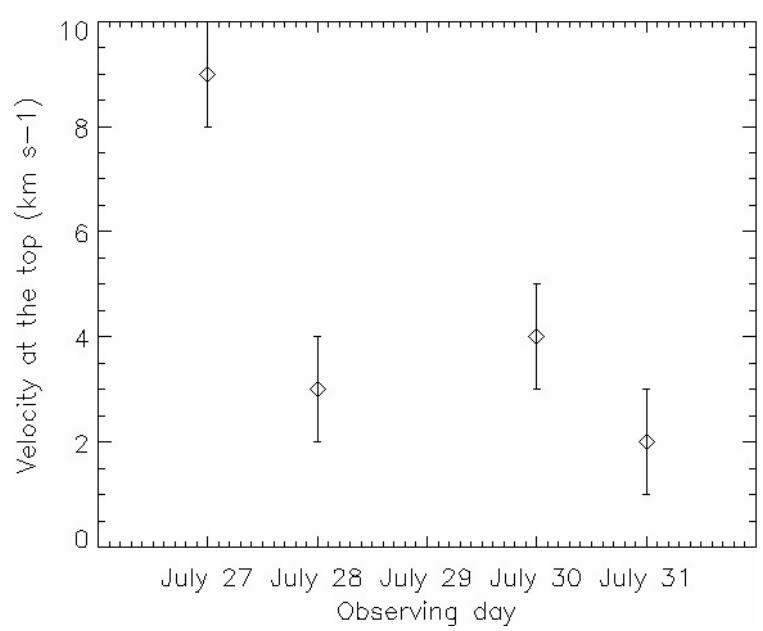

Fig. 16. Plot of the maximum velocities measured at the top of AFS during each observing day.

the magnetic flux emergence as more and more thin flux tubes rise towards higher atmospheric layers.

Moreover the observed asymmetries between the preceding and following parts of the active region confirm the model of a thin flux tube rising through a passive medium under the influence of magnetic, pressure, rotational and drag forces (Caligari et al. 1995). More precisely, the first asymmetry concerns the greater extension of the $\mathrm{p}$ sunspot $(\sim 26000 \mathrm{~km}$ in diameter on July 28) with respect to the f sunspot $(\sim 21000 \mathrm{~km}$ on the same day). A second asymmetry concerns the greater velocity towards the West in the preceding polarity with respect to that towards the East measured in the following polarity. Thirdly, the plasma motions at the ends of the AFs show an asymmetry consisting of a stronger downflow at the following footpoints than at the preceding ones. This asymmetry can be interpreted as due in part to an inclination of the emerging AFs. It has been widely described by Caligari et al. (1995), as a consequence of the conservation of angular momentum during the flux tube rise: whenever a mass element of a flux tube goes up in the convection zone, the Coriolis force imparts an acceleration opposite to the direction of rotation so that the angular momentum is conserved. The angular momentum conservation tends, therefore, to cause a relative motion of the crest of the tube with respect to the valley in the direction opposite to the solar rotation. The speed of this relative motion is larger the higher the level reached by the crest of the tube and the deeper the anchored parts penetrate into the overshoot region.
This relative motion tends to make the following side closer to the vertical than the preceding side. The average value of the difference of inclination between the $\mathrm{p}$ - and f-wing for the first day of observation is $\sim 20$ degrees, while it seems to decrease on the following day.

On the other hand, we must also take into account that Cauzzi et al. (1996) determined the photospheric vertical velocities in the magnetic elements of the preceding and following polarities of young active regions, and found a higher downflow in the p-side than in the f-side. This result seems to be in contrast with the asymmetries we have found in the legs of the AFs. Nevertheless, before drawing a conclusion, we have to consider the three possibilities listed above: the first possible cause of the downflow is the Coriolis force acting on the rising flux tube, which tends to create a counterflow (i.e. a flow along the field lines opposite to the direction of rotation); the second cause, that is the instability-related sliding flow, is p-f symmetric, so it cannot give a contribution to any asymmetric vertical motion; the third cause is related to the remnant longitudinal motion in the original mechanical equilibrium, which requires a flow along the field lines compensating the magnetic flux stresses so that the velocity increases with the field strength. Therefore the behaviour of the longitudinal motion in the flux tube will be determined by the competition between the effect of the Coriolis force and the original flow. In particular, the condition of higher p-side downward velocity is met when the initial flux tube velocity is high enough for the Coriolis force not to have time to induce a change of sign in the velocity during the flux tube rising. This in turn depends on the values of the magnetic field strength. Therefore the difference between our results and those reported by Cauzzi et al. (1996) might be due to differences in the initial magnetic field strength (see also Fan et al. 1993, 1994). Anyway, it is interesting that in both studies the asymmetry decreases as the region ages.

Finally, since symmetric sliding flows were investigated many times in the past (see, for example, the "leaky bucket" model (Schmieder et al. 1991) or the simple dynamical model of emerging arches (Mein et al. 1996)), we give an estimate of the competition between the AFS plasma asymmetric motion due to Coriolis force and the symmetric sliding motion, by means of the inverse of the Rossby number (see, e.g., Stix 1991, p. 223). More precisely, the Rossby number is: $R_{0}=v /(2 \Omega l)$, where $v$ and $l$ are the typical velocity and scale length of the system and $\Omega$ is the average rotation rate. If we substitute the 


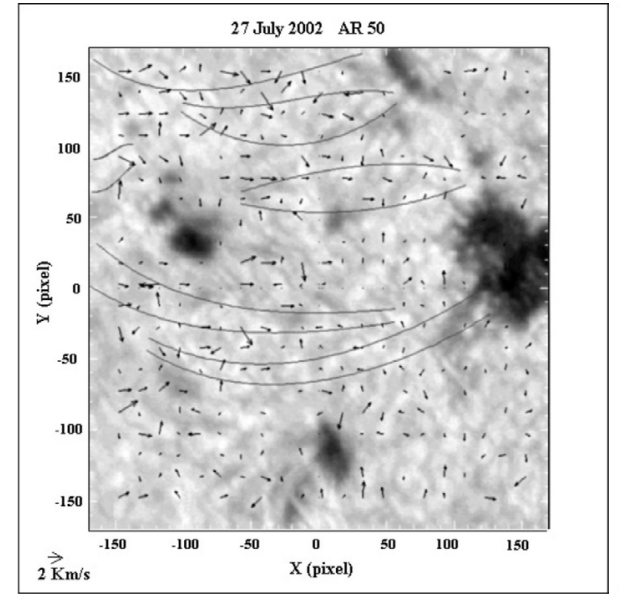

(a)

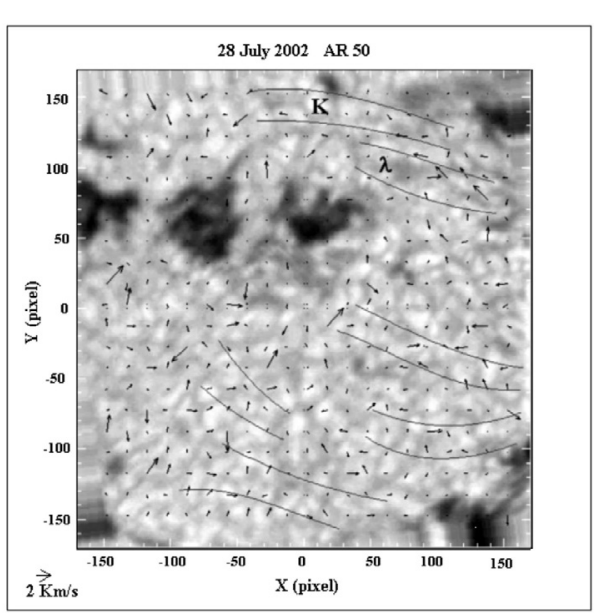

(b)

Fig. 17. a) Horizontal velocity map obtained using the broad band images acquired on July 27; b) Horizontal velocity map obtained from the broad band images acquired on July 28. The contours plotted over the images represent the AFs observed in H $\alpha$. The field of view is $\sim 2400 \times 2400 \mathrm{~km}^{2}$. In all images North is at the top and West on the right.

values $\Omega=3 \times 10^{-6} \mathrm{~km} \mathrm{~s}^{-1}$ and $l=1.4 \times 10^{4} \mathrm{~km}$ (the average value of the arches observed), we find that in the emerging AFS the inverse of the Rossby number can have values between $10^{-1}$ and $10^{-2}$, corresponding to upward velocity values between 1 and $10 \mathrm{~km} \mathrm{~s}^{-1}$. Therefore it seems that a counterflow due to the Coriolis force in the emerging flux tubes can significantly compete with the sliding motions only when the upward velocity decreases to values below $1 \mathrm{~km} \mathrm{~s}^{-1}$ (not observed in our AFS), or when the arches are characterized by a greater length. This implies that the Coriolis force can account for the higher downward velocity observed in the following side if the entire size of the emerging flux tubes is considered, not only that of the part visible in chromosphere.

We would like to conclude that all the results obtained in this analysis could be considered as indicative of the phenomena occurring during the formation of an active region which undergoes a complete evolution. Therefore it would be very important to verify whether an active region rapidly decaying in a few days is characterized by the same values of upward velocities in the arches forming the AFS and, more importantly, whether these arches show the same decreasing upward velocity we have observed in NOAA 10050 as the active region gets older.

Acknowledgements. The authors wish to thank E. Catinoto and S. Sciuto for their kind support and cooperation in getting white light and $\mathrm{H} \alpha$ images at the INAF-Catania Astrophysical Observatory. The warm and fruitful help by the THEMIS resident staff is acknowledged. The authors also wish to thank the teams of the EIT and MDI instruments on board SOHO, as well as the team of TRACE, for participating in the coordinated campaign of observations which allowed this investigation. A special thank to A. Kosovichev, for arranging and providing the MDI data cubes which were used to follow the behaviour of the magnetic field during the evolution of the NOAA 10050 active region. The help of F. Auchere in planning an extra set of EIT synoptic observations during the campaign and providing the relevant data is also acknowledged. The authors thank P. Mein for his comments and suggestions which led to a sounder version of the manuscript. This work was supported in part by the Italian
Ministry of Education, University and Research, and in part by the Agenzia Spaziale Italiana (contract ASI I/R/32/00).

\section{References}

Alissandrakis, C. E., Tsiropoula, G., \& Mein, P. 1990, A\&A, 230, 200 Arnaud, J., Mein, P., \& Rayrole, J. 1998, in a Crossroads For European Solar and Heliographic Physics, Tenerife, ESA SP-417, 213

Caligari, P., Moreno-Insertis, F., \& Schussler, M. 1995, ApJ, 441, 886

Cauzzi, G., Canfield, R. C., \& Fisher, G. H. 1996, ApJ, 456, 850

Cavallini, F. 1998, A\&A, 128, 589

Chae, J. 2001, ApJ, 560, L95

Contarino, L., Romano, P., Yurchyshyn, V. B., \& Zuccarello, F. 2003, Sol. Phys., 216, 173

Delaboudinière, J.-P., Artzner, G. E., Brunaud, J., et al. 1995, Sol. Phys., 162, 291

Fan, Y., Fisher, G. H., \& De Luca, E. E. 1993, ApJ, 405, 390

Fan, Y., Fisher, G. H., \& McClymont, A. N. 1994, ApJ, 436, 907

Handy, B. N., Acton, L. W., Kankelborg, C. C., et al. 1999, Sol. Phys., 187,229

Howard, R. F. 1991, Sol. Phys., 134, 233

Kitai, R., Funakoshi, Y., Ueno, S., Sano, S., \& Ichimoto, K. 1997, PASJ, 49, 513

Mein, P., Demoulin, P., Mein, N., et al. 1996, A\&A, 305, 343

Malherbe, J. M., Schmieder, B., Mein, P., et al. 1998, Sol. Phys., 180, 265

Moreno-Insertis, F. 1997, Mem. Soc. Astron. It., 68, 429

Parker, E. N. 1993, ApJ, 408, 707

Petrovay, K., Brown, J. C., van Driel-Gesztelyi, L., et al. 1990, Sol. Phys., 127, 51

Priest, E. R. 1987, Solar Magnetohydrodynamics (Dordrecht: Reidel) Scherrer, P. H., Bogart, R. S., Bush, R. I., et al. 1995, Sol. Phys., 162, 129

Schmieder, B., Raadu, M. A., \& Wiik, J. E. 1991, A\&A, 252, 353

Stix, M. 1991, The Sun: An Introduction (Berlin: Springer)

Strous, L. H., Scharmer, G., Tarbell, T. D., Title, A. M., \& Zwaan, C. 1996, A\&A, 306, 947

Strous, L. H., \& Zwaan, C. 1999, ApJ, 527, 435

van Driel-Gesztelyi, L., \& Petrovay, K. 1990, Sol. Phys., 126, 285

van Driel-Gesztelyi, L. 2002, IAU Coll., 188, ESA SP-505, 113

Zuccarello, F., Contarino, L., Romano, P., et al. 2002, INAF-OACt Internal report N. 1

Zwaan, C. 1985, Sol. Phys., 100, 397 